\title{
CLINICAL AND PROGNOSTIC IMPACT OF CHANGES IN THE TNM SYSTEM OF THE AMERICAN JOINT COMMITTEE FOR CANCER, EIGHTH EDITION, IN PATIENTS WITH BREAST CANCER
}

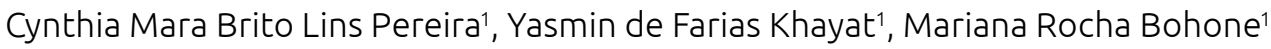 \\ ${ }^{1}$ Centro Universitário do Pará - Belém (PA), Brazil.
}

In Brazil, breast cancer is the first among the most prevalent malignancies in women, without considering non-melanoma skin cancer. However, in spite of the high number of deaths caused by breast cancer, there has been a great reduction in mortality rates and greater survival of patients with metastatic disease in the last decades. Such improvements are related to advances in treatment and greater knowledge about the biology of breast cancer. The American Joint Committee for Cancer (AJCC) cancer staging system is one of the important tools for doctors, and helps to predict disease progression and make therapeutic decisions. Therapeutic planning and prognosis of patients is possible through staging. Since the publication of the first edition of the Cancer Staging Manual, AJCC has insisted on seeing anatomical information. TNM staging (T: tumor; N: lymph nodes; M: metastasis). Limitations regarding this staging method were evidenced, as it is based only on anatomy and does not take biological factors into account. Through immunohistochemical study, breast cancer is subdivided into different molecular subtypes. When considering the modifications of the new edition of the TNM/AJCC system with respect to the prognostic and predictive factors of cancer, there may be a reclassification of patients, leading to a more reliable approach to their real disease condition. Objective: To analyze the impact generated by the update of the TNM/AJCC staging system (eighth edition), in the classification of patients with breast cancer seen at Hospital Ophir Loyola, a referral oncology hospital in the city of Belém, state of Pará, in 2018. Method: 176 medical records of patients undergoing treatment at Hospital Ophir Loyola, in 2018, were analyzed, which had information on the staging of the seventh edition and with immunohistochemical results. Result: $61.93 \%$ were between $40-60$ years old, $46.2 \%$ were from the capital. Regarding the stage of diagnosis according to the 7th edition, 23 patients (13\%) were in stage I, 66 cases (37.5\%) in stage II, and the vast majority, totaling 77 cases (43.8\%), in stage III. In addition, there were 03 cases (1.7\%) in stage 0 (zero), and 07 cases (4\%) in stage IV. There was a change in disease staging for $60.8 \%(107 / 176 ; 95 \% \mathrm{CI}$ $53.4-67.7)$ of the cases, $36.5 \%$ (39/107; 95\%CI 28.0-45.9) of these cases were upstaged, and in the others (63.5\%, 68/107; 95\%CI 54.1-72.1), the change was to a lower prognostic category (down-staged). There was a significant increase in the proportion of cases staged in 2018 as IB and a significant reduction in cases staged by the most recent criterion such as IIB and IIIA ( $\mathrm{p}<0.0001)$. Conclusion: the changes to new staging have shown to be more effective on the behavior of the tumor, helping in therapeutic decisions. 